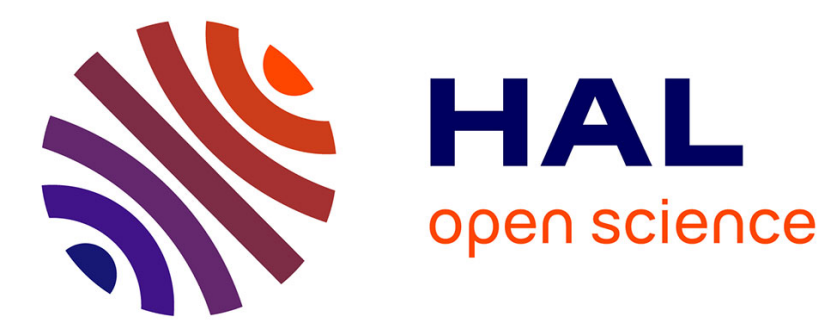

\title{
Pour une histoire des humanités numériques
}

Aurélien Berra

\section{To cite this version:}

Aurélien Berra. Pour une histoire des humanités numériques. Critique, 2015, Des chiffres et des lettres : les humanités numériques, 819-820, pp.613-626. halshs-01182509

\section{HAL Id: halshs-01182509 \\ https://shs.hal.science/halshs-01182509}

Submitted on 15 Oct 2015

HAL is a multi-disciplinary open access archive for the deposit and dissemination of scientific research documents, whether they are published or not. The documents may come from teaching and research institutions in France or abroad, or from public or private research centers.
L'archive ouverte pluridisciplinaire HAL, est destinée au dépôt et à la diffusion de documents scientifiques de niveau recherche, publiés ou non, émanant des établissements d'enseignement et de recherche français ou étrangers, des laboratoires publics ou privés. 
Aurélien Berra, "Pour une histoire des humanités numériques", Critique, 819-820, 2015, p. 613-626. - Version d'auteur.

\section{POUR UNE HISTOIRE DES HUMANITÉS NUMÉRIQUES}

À propos de :

Willard McCarty, Humanities Computing, Houndmills, Basingstoke, Palgrave Macmillan, 2005

Fred Turner, Aux sources de l'utopie numérique. De la contre-culture à la cyberculture, traduit par Laurent Vannini, Caen, CEF éditions, 2012 (From Counterculture to Cyberculture: Stewart Brand, the Whole Earth Network and the Rise of Digital Utopianism, Chicago, University of Chicago Press, 2006)

«Solon, Solon, vous autres Grecs êtes toujours des enfants; vieux, un Grec ne peut l'être. » Sur ce, Solon s'enquit : «Que veux-tu dire par là? " Et le prêtre de répondre : "Jeunes, vous l'êtes tous par l'âme, car vous n'avez en elle aucune vieille opinion transmise depuis l'antiquité de bouche à oreille, ni aucun savoir blanchi par le temps ${ }^{\mathrm{I}}$."

Les humanités numériques ont une sulfureuse pertinence pour notre temps. Parce que nous recourons à des moyens de communication et d'archivage nés il y a quelques décennies seulement, parce que le fonctionnement public et privé de nos sociétés repose désormais sur le traitement de jeux de données, nous reconnaissons aux technologies informatiques une importance en tant que phénomène social, politique, économique et culturel. Ces transformations, subtiles ou subites, de nos façons d'être ont-elles des conséquences appréciables sur nos façons de connaître? La question prêterait à sourire dans les disciplines où l'on ne peut guère oublier les conditions de la recherche qui se fait aujourd'hui, à savoir l'outillage, les infrastructures, des bases de données d'une taille sans précédent, les algorithmes, les échanges presque instantanés. Dans les sciences humaines et sociales, les chercheurs estiment souvent que rien d'essentiel n'a encore été acquis, que le saut qualitatif est un argument de publicité, voire de propagande. Peut-on faire confiance à ceux qui se rangent sous la bannière des Digital Humanities - suspects de revendiquer leur adéquation à une

I. Platon, Timée, 22 a-b (trad. Luc Brisson et Michel Patillon, Paris, GF-Flammarion, p. I07). 
révolution numérique, quoique parfois sommés de sauver des traditions d'étude menacées par le discrédit et la pénurie?

Ce scepticisme s'enracine en partie dans le terreau de nos représentations sociales contrastées de l'informatique. D'un côté, le progrès et une promesse de libération; de l'autre, la grisaille bureaucratique, le cheval de Troie d'une administration stérile, la menace d'un élément inhumain inoculé dans nos systèmes de savoir. Pour éclairer les regards jetés sur nos pratiques et nos cultures savantes numériques, il est fondamental de disposer d'une perspective historique.

\section{Étapes historiques}

En quête de légitimité, les humanités numériques font valoir qu'elles ont une histoire déjà longue. Au commencement étaient les œuvres de Thomas d'Aquin, puis vers I949 vint Roberto Busa, son commentateur jésuite et notre père fondateur, qui alla trouver le patron de l'International Business Machines Corporation (IBM) et mit en fiches perforées l'Opus Thomisticum pour en élaborer la concordance. Ainsi débute le récit canonique proposé dans un companion book ${ }^{2}$, dans cent billets de blog et dans les pages de Wikipédia.

Les périodisations habituelles s'accordent sur des césures majeures. Les grandes évolutions des machines sont des bouleversements techniques et culturels bien connus. Des années I940 aux années I980, l'ère des mainframe computers, infrastructures colossales placées au centre de laboratoires principalement militaires et industriels, voit l'élaboration progressive de logiciels standards ne dépendant pas du hardware. Au milieu des années I980, les ordinateurs dits personnels, qui deviendront de plus en plus portables, diffusent les interfaces graphiques, l'hypertexte et le courrier électronique. À partir des années I990, les ordinateurs fonctionnent en réseau : l'Internet et le Web, à la faveur surtout de la miniaturisation des terminaux et des interfaces tactiles et vocales, commencent à donner une réalité aux rêves d'informatique ubiquitaire, les puces omniprésentes densifiant la doublure informationnelle du monde. Cela n'est possible que grâce à l'accroissement exponentiel de la puissance de calcul et des capacités de stockage - des cartes perforées aux bandes magnétiques, puis aux disques électro-magnétiques ou à mémoire flash.

Dans ce cadre chronologique, nos aperçus des humanités numériques signalent des projets remarquables, tout en retraçant le développement institutionnel du champ, c'est-à-dire l'apparition - et la disparition - des revues, des centres de recherche et des institutions transdisciplinaires, des associations professionnelles, des diplômes et des formations, ainsi que l'extension des collaborations entre métiers 3 .

2. Susan Schreibman, Raymond Siemens et John Unsworth (éd.), A Companion to Digital Humanities, Oxford, Blackwell, 2004, http://www.digitalhumanities.org/companion. L'ouvrage doit être bientôt mis à jour : il sera intéressant d'en mesurer l'évolution. 
L'Index de Roberto Busa correspond à une phase initiale consacrée aux travaux de philologie et de stylistique devenus réalistes : concordances, dictionnaires, approches quantitatives des questions de style et d'attribution. En 1966, Joseph Raben fonde la revue Computers and the Humanities. À cette époque, des centres d'étude se créent à Cambridge, Tübingen ou Louvain. En I972, une institution professionnelle internationale voit le jour, l'Association for Literary and Linguistic Computing; son Bulletin initial devient en 1986 la revue Literary and Linguistic Computing, renommée en 2015 Digital Scholarship in the Humanities. Les archives et les bases de données textuelles se développent, comme l'Oxford Text Archive (1976) et le Thesaurus Linguae Graecae (1972). La Text Encoding Initiative propose depuis 1987 l'élaboration collaborative de normes d'encodage savant des textes et des documents. La même année, Willard McCarty ouvre la liste de discussion électronique Humanist. En I990, la Bryn Mawr Classical Review commence à diffuser par le même canal des comptes rendus de monographies scientifiques. On met en ligne les " archives " de quelques grands auteurs du patrimoine mondial. Au début du Xxi ${ }^{\mathrm{e}}$ siècle, des diplômes et des cursus se forment, au Royaume-Uni, aux États-Unis et au Canada, puis dans divers pays du monde, tandis que les écoles d'été et d'autres événements moins formels demeurent les modes de transmission des connaissances les plus caractéristiques. Des institutions continentales et mondiales se mettent en place, dont l'Alliance of Digital Humanities Organizations (ADHO) en 2006.

Au fil de ce demi-siècle, les dénominations ont varié : Literary and Linguistic Computing, Humanities Computing et, depuis une quinzaine d'années, Digital Humanities, avec leurs calques et leurs variantes dans d'autres langues, moins bien répertoriés (informatique littéraire, informatica umanistica, digitale Geisteswissenschaften, etc.). Certaines ont clairement des portées différentes et ne désignent pas des vagues successives. Toutes portent la trace des degrés d'autonomie qu'on reconnaît à ces activités et des relations négociées avec les disciplines constituées.

Ces étapes attestent la construction d'un milieu, en privilégiant les " premières fois " et les projets encore actifs. Elles pointent vers des questions décisives, comme le partage et la pérennité des données scientifiques, la

3. Pour des historiques plus développés, voir Willard McCarty, "Humanities Computing ", dans Miriam Drake (éd.), Encyclopedia of Library and Information Science, 2003, Dekker, New York, p. I224-I 235 ; Susan Hockey, "The History of Humanities Computing ", dans Schreibman et al. 2004, p. 3-19; Gregory Crane, "Classics and the Computer : An End of the History ", dans Schreibman et al. 2004, p. 46-55; Lou Burnard, "Du literary and linguistic computing aux digital humanities: retour sur 40 ans de relations entre sciences humaines et informatique ", dans Pierre Mounier (éd.), Read/Write Book 2. Une introduction aux humanités numériques, Marseille, OpenEdition Press, 2012, p. 45-58 et http://books.openedition.org/oep/242 ; Olivier Le Deuff et Frédéric Clavert, « Petite histoire des humanités digitales ", dans Olivier Le Deuff (éd.), Le Temps des humanités digitales, Limoges, FYP Éditions, 20I4, p. I5-3I. Pour une perspective critique plus riche, voir Teresa Numerico, Domenico Fiormonte et Francesca Tomasi, L'Umanista digitale, Bologne, Il Mulino, 20 Io. 
collaboration entre individus et entre communautés, la formation. Implicitement, elles font apercevoir des problèmes, tels le traitement des images et des artefacts, la question du multilinguisme ou les dominantes et les amnésies disciplinaires.

Néanmoins, une véritable historiographie nous fait défaut. L'histoire politique ne se réduit plus depuis longtemps à une histoire événementielle, ni l'histoire des sciences à la liste ordonnée des découvertes et des découvreurs. Mais selon quels critères sélectionner les éléments pertinents ? Où commencer? Si la gageure est de donner un sens aux pratiques et aux propositions scientifiques, il faudrait les réintégrer dans une histoire des outils, des techniques - façons de faire efficaces, extensions des capacités physiques, cognitives et sociales, sciences appliquées -, ainsi que des imaginaires culturels associés. Cela revient à cerner la place de la technologie dans la société et dans la recherche, à travers l'histoire des automates, des dispositifs savants de manipulation des données (dont le codex, cette " machine à penser " selon l'expression de I. A. Richards, bien avant les fichiers inscrits dans des métaux conducteurs), des appareils de calcul et, bien sûr, des formalisations théoriques mettant en communication la pensée et le mécanique (notamment la "machine » d'Alan Turing et la cybernétique de Norbert Wiener). On le voit, ce chantier est vaste et à peine ouvert.

Roberto Busa, décédé en 20 I , a écrit qu'il voyait dans l'avènement du traitement automatique des langues, et plus largement dans les humanités numériques, "le doigt de Dieu ", un signe de la providence 4 . Victimes de l'illusion d'un déterminisme technologique, nous courons le risque de laïciser cette métaphore. À l'inverse, il s'agit ici d'insister sur l'historicité de ce champ et d'examiner pourquoi écrire l'histoire des Digital Humanities est une entreprise complexe. Le récit standard est aveugle à la diversité non seulement des domaines, mais aussi des pays et des zones linguistiques et culturelles. Notre vulgate n'est pas satisfaisante.

Les discours portant sur "le " numérique ravivent, consciemment ou non, une notion réductrice du progrès. Positivisme, scientisme, désir indu de mimer les sciences exactes, telles seraient les tares de toute méthode recourant à la discrétisation et à la quantification. Dans une version résignée de cette vision, les Digital Humanities seraient une destruction créatrice inéluctable. Ces réceptions angoissées, récurrentes, sont elles-mêmes passibles d'une histoire.

Il est clair, en revanche, que ce mouvement tire son énergie d'un esprit d'invention et d'adaptation. Même l'application de méthodes plus ou moins éprouvées à des objets d'enquête traditionnels recèle un potentiel heuristique. Alors que nos institutions sont souvent peu propices à un renouvellement rapide des savoirs et des pratiques, nous vivons encore l'âge des prototypes. Un historien a d'ailleurs suggéré de faire droit au temps des démonstrations : les sociétés savantes du XVIII ${ }^{\mathrm{e}}$ siècle n'ont-elles pas posé les fondements des

4. Roberto Busa, «Foreword: Perspectives on the Digital Humanities », dans Schreibman et al. 2004, p. XVI-XXI. 
sciences modernes en faisant se côtoyer le sérieux et le douteux, le spectacle technique et l'expérience scientifique, sous le signe de l'expérimentation 5 ?

En réalité, la valorisation de l'essai s'accompagne au moins autant de réflexivité critique que d'euphorie ludique. La question de l'innovation technologique semble cardinale dans l'identité de praticiens qui cherchent la nouveauté par des moyens inexplorés. Loin du modèle des mises à jour numérotées de logiciels - qui sont le reflet d'une relation particulière à la temporalité, c'est-à-dire au travail collectif et au commerce de produits industriels selon des cycles courts -, aux yeux de nombreux auteurs, la valeur fondamentale des méthodes numériques est un questionnement permanent concernant les outils et les fins du travail savant ${ }^{6}$.

\section{La dimension critique}

L'histoire elle-même explique certaines des critiques formulées de l'extérieur du champ. Il n'est pas rare que des chercheurs expérimentés se désintéressent par principe des propositions de tard-venus qui commenceraient seulement au $\mathrm{XxI}^{\mathrm{e}}$ siècle à utiliser l'informatique, alors que des branches de l'histoire ou de la linguistique, par exemple, ont développé des outils avant la popularisation, ou la prolétarisation, entraînée par le Web. Plus couramment, on assimile les projets informatisés à certains aspects de la Toile : valorisation de l'éphémère et présentisme, pérennité incertaine des productions, nivellement dans une culture numérique commune incarnée par les réseaux sociaux, dont on efface la diversité des usages. Des discussions argumentées seraient plus utiles.

La critique interne mérite ici davantage d'attention. Un nombre croissant d'interventions, orales et écrites, réfléchissent à l'obsolescence des techniques ou à la difficulté de la transmission des expériences et des compétences entre générations et entre équipes. Comment faire des humanités numériques un courant pleinement actuel, en rapport avec les pensées les plus vives des sciences humaines et sociales? Les débats sont ouverts 7.

Le livre publié en 2005 par Willard McCarty, sobrement intitulé Humanities Computing, est probablement la réflexion la plus profonde et la plus ample sur ces sujets. Méditation personnelle sur l'histoire d'une communauté, elle se présente comme une synthèse et un acte de fondation pour une épistémologie critique. L'auteur analyse le devenir des genres de production savante et le statut des disciplines; il montre la computer science comme une entreprise intellectuelle ouverte et plurielle; il propose enfin un

5. Tom Scheinfeldt, "Where's the Beef? Does Digital Humanities Have to Answer Questions? ", dans Gold 2012 (voir référence infra), p. 56-58.

6. Julia Flanders, "The Productive Unease of 2 Ist-century Digital Scholarship ", Digital Humanities Quarterly, 3, 3, 2009, http://www.digitalhumanities.org/dhq/vol/3/3/000055/ $000055 \cdot \mathrm{html}$.

7. Matthew Gold, Debates in the Digital Humanities, Minneapolis, University of Minnesota Press, 20 I 2, http://dhdebates.gc.cuny.edu/debates. Sur les thèmes mentionnés, voir les contributions de Bethany Nowviskie, "Eternal September of the Digital Humanities" (p. 243-246), et Alan Liu, «Where Is Cultural Criticism in the Digital Humanities? " (p. 490509). 
programme et appelle à l'action collective. C'est sur ce dernier aspect que nous insisterons. McCarty envisage les humanités numériques comme un espace de dialogue, une zone d'échange dans laquelle se rencontrent les données et les méthodologies des différentes traditions savantes. Pour chaque praticien, elles sont aussi l'occasion d'une expérience réflexive instrumentée, dans la mesure où la formalisation impose une transformation des catégories reçues. Pour illustrer le concept central de modélisation, son exemple est une enquête sur la notion de personnage dans les Métamorphoses d'Ovide, qu'il a menée à travers un encodage extrêmement détaillé du texte.

Cette activité critique essentielle contraint-elle à un perpétuel recommencement, chaque chercheur et chaque équipe récapitulant dans la genèse des projets la phylogenèse de ce domaine d'étude? L'enjeu même de l'institutionnalisation, de plus en plus rapide, est de permettre la création d'une culture. Une histoire partagée en serait l'un des piliers.

Avant de mentionner de quelle façon cet objectif a été revendiqué depuis quelques années, arrêtons-nous sur un facteur important de cette culture, l'ambivalence idéologique des technologies numériques. Dans un ouvrage paru en 2006, Fred Turner en esquisse une véritable contre-histoire, au fil du parcours exceptionnellement riche de Stewart Brand, créateur du Whole Earth Catalog, que certains voient comme l'ancêtre de papier du Web, et cofondateur de la première communauté virtuelle, le WELL, du Global Business Network, qui a permis aux milieux d'affaires d'appliquer à l'économie la métaphore du réseau, ainsi que du magazine technophile Wired. Il fait ainsi ressortir une trame complexe mais non contradictoire, où apparaît le rôle des utopies, depuis les rêves de communauté et les techniques de libération des années I960 jusqu'à leur reconversion dans les forums en ligne et dans les idéaux affichés par les grandes entreprises comme Apple. L'injonction paradoxale à tous penser autrement - Think Different - ne serait pas une hypocrisie lucrative, mais l'un des signes de notre temps.

Il n'entre pas dans le propos de Turner de relier ce mariage historique de l'utopie et de l'opportunisme entrepreneurial aux évolutions du monde de la recherche ${ }^{8}$. Toutefois, son récit met au jour les origines d'un éthos hybride, ou d'un spectre de réactions à la question technologique chez les praticiens : hackers et érudits, contestataires motivés par le détournement des usages prévus et fondateurs de standards ou de bonnes pratiques, expérimentateurs et administrateurs de la science. Les industries créatives et les start-ups du Web sont une version extrême d'une culture fondée sur le " mode projet " et une collaboration dite horizontale. Ce modèle est spécialement subversif pour les mondes savants, leur rapport au temps et leurs valeurs parfois séculaires. Il peut suggérer de rendre explicite le rôle de l'esthétique, des affects et du plaisir dans l'expérience de l'interprétation, pour rendre justice à l'aesthesis, cruciale dans les sciences humaines, face à la mathesis constitutive de nos conceptions de la science 9 . D'un point de vue sociologique,

8. Aurélien Berra et Pierre Mounier, «DH EHESS : Aux sources de l'utopie numérique. Compte rendu ", Philologie à venir, 25.02.20I4, http://philologia.hypotheses.org/I $37 \mathrm{I}$ (résumé et enregistrement d'une séance de séminaire). 
ces dangereuses liaisons nous rappellent que les savants sont aussi membres d'une société qui se numérise, non seulement par leurs pratiques privées, mais aussi par leur appartenance à des institutions qui ont à se réinventer selon des modèles managériaux de gestion des ressources. Les humanités numériques succombent-elles aux sirènes du cool et à la tentation d'une distinction par la technologie, spécialement forte dans des disciplines en déclin ? L'hypothèse est superficielle ${ }^{\mathrm{IO}}$. Cependant, tous les digital humanists ne sont pas des épistémologues ni des algorithmiciens - et la variété des compétences et des sensibilités fait l'intérêt de ce milieu.

\section{Les histoires parallèles des humanités numériques}

En suivant la trajectoire d'un acteur, Turner fait apparaître une continuité entre divers états d'un système sociotechnique et culturel. Ce choix procède également d'une nécessité historiographique : la profondeur de l'analyse dépendait de la précision documentaire. On pourrait comparer le problème avec les expérimentations de l'historia en Grèce ancienne. Les considérations générales plongent des racines aussi bien dans les registres et les histoires locales que dans les témoignages. Outre le travail des sources, Hérodote, Thucydide ou les historiens d'Alexandre empruntent la voie de l'expérience directe, en intégrant diversement la subjectivité, les conflits et les stratégies comme l'une des réalités de l'histoire. Willard McCarty répond à cette double nécessité en nourrissant son panorama d'un parcours individuel et en proposant un agenda à ses lecteurs. Y figure en bonne place l'incitation à écrire une histoire " authentique".

Depuis quelques années, on observe en effet un regain d'intérêt pour le développement historique, les succès et les échecs de cette communauté hétérogène. Ce désir d'historiographie est collectif. La volonté de documenter les projets, les méthodes et les pratiques du passé s'exprime ainsi dans la préparation d'un "groupe d'intérêt spécial » au sein de l'association internationale $\mathrm{ADHO}$, dont la première tâche devrait être d'inventorier et de rassembler les fonds documentaires. Plus encore, des interrogations pressantes émanent des institutions qui incarnent l'internationalisation en cours : citons le groupe Global Outlook::Digital Humanities, qui a été en 2012 le premier groupe de travail officiel d'ADHO, et les associations nationales ou linguistiques qui se multiplient depuis 20I I, dont la dernière en date est Humanistica, l'association francophone des humanités numériques, fondée en 20 I 4.

Cette tendance répond autant à un besoin épistémologique - rétablir une histoire plus pertinente localement et, par conséquent, globalement - qu'à des processus de construction identitaire. Ce sont les histoires qui sont le matériau premier d'une histoire commune. Telle est la prémisse du projet

9. Johanna Drucker, SpecLab. Digital Aesthetics and Speculative Computing, Chicago, University of Chicago Press, 2009, où l'on trouve l'histoire réflexive d'un centre de recherche.

ı. Selon Alan Liu, la catégorie du cool ne recouvre pas seulement un style, mais potentiellement une résistance à l'idéologie de l'information à l'intérieur même des technologies de l'information: Alan Liu, The Laws of Cool. Knowledge Work and the Culture of Information, Chicago, University of Chicago Press, 2004. 
Hidden Histories, qui s'appuie sur la méthodologie de l'histoire orale pour constituer une mémoire publique à travers des entretiens avec de grandes personnalités des Digital Humanities ${ }^{\text {II }}$. Ce projet est anglophone, mais prévoit d'élargir les aires culturelles concernées, voire de donner la parole aux acteurs ordinaires. L'extension paraît décisive, si l'on veut comprendre les usages des technologies informatiques dans des contextes extrêmement différents. Une telle perspective prend acte du fait que toute épistémologie est historique et rejoint les ambitions d'une anthropologie des pratiques savantes ${ }^{\mathrm{I2}}$.

Décrire ce qui est et écrire ce qui a été sont deux mouvements solidaires. McCarty se réfère à la notion de thick description introduite par Clifford Geertz et note qu'un point de vue global ne peut être constitué que par la mise en relation d'histoires nationales, linguistiques, sociales et disciplinaires jamais tout à fait synchrones. Les locuteurs natifs de l'anglais, lingua franca des Digital Humanities, y perdront peut-être en pouvoir, mais y gagneront beaucoup en savoir. Les membres des autres communautés linguistiques, intégrés ou non aux institutions internationales anglophones, ont à mener leur travail de mémoire. L'horizon commun serait finalement une histoire comparée, éclairée par l'épistémologie comparative de l'histoire des sciences contemporaine.

La question est résurgente et il serait inexact de dire que nous n'avons pas d'exemple. En 1999, McCarty lui-même avait eu des échanges sur la liste Humanist avec Tito Orlandi, le premier théoricien et praticien de l'Informatica Umanistica italienne, qui s'indignait que ses idées publiées en italien soient totalement ignorées. Domenico Fiormonte, analyste de la domination scientifique anglophone et partisan d'un réel multiculturalisme, est d'ailleurs l'un des élèves d'Orlandi ${ }^{13}$. Du point de vue des disciplines, les productions d'un réseau franco-italien d'historiens médiévistes ${ }^{\mathrm{I} 4}$ montrent bien ce que peut apporter un premier effort de synthèse. De fait, l'interdisciplinarité naturelle du champ impose de retracer des évolutions partiellement indépendantes. Pour comprendre les humanités numériques, il faut - c'est un truisme - saisir ce qu' elles doivent aux inflexions des sciences humaines et sociales, autant que ce qu' elles doivent à l'informatique.

Un récent projet d'histoire comparée des " humanités " inclut désormais des contributions consacrées aux Digital Humanities ${ }^{15}$. La question classique de leur statut est avant tout liée à leur devenir institutionnel. Est-il justi-

I I. Julianne Nyhan, Andrew Flinn et Anne Welsh, "Oral History and the Hidden Histories Project : Towards Histories of Computing in the Humanities ", Digital Scholarship in the Humanities, 30, I, 20 I 5, p. 7185. Voir également https://hiddenhistories.omeka.net.

I 2. Christian Jacob (éd.), Lieux de savoir. I. Espaces et communautés et Lieux de savoir. II. Les mains de l'intellect, Paris, Albin Michel, 2007 et 20 I I.

I3. Domenico Fiormonte, "Towards a Cultural Critique of the Digital Humanities ", Historical Social Research/Historische Sozialforschung, 20 I 2, p. 5976.

I4. Jean-Philippe Genet et Andrea Zorzi (éd.), Les Historiens et l'informatique. Un métier à réinventer, Rome, École française de Rome, 20 I I.

I 5. Rens Bod, A New History of the Humanities. The Search for Principles and Patterns from Antiquity to the Present, traduit par Lynn Richards, Oxford, Oxford University Press, 2013. Bod Rens, Jaap Maat et Thijs Weststeijn (éd.), The Making of the Humanities, Amsterdam 
fié de les doter de postes et de cursus indépendants? Toute une gamme de relations sont possibles avec les programmes que constituent les disciplines : traitement de données conçu comme une prestation de services, collaboration effective, autonomisation d'un nouveau courant ou d'une nouvelle discipline. Il suffit de penser à la linguistique, dont des pans entiers ont été renouvelés par les approches computationnelles des corpus, ou aux sciences de l'information et de la communication, dont le domaine propre s'est récemment établi. Dans ces deux cas, une relation naturelle existe avec les humanités numériques, mais elle est fluctuante et fréquemment tendue. Cette histoire a aussi pour enjeux des territoires.

Usagers de machines et de logiciels qui sont autant de "boîtes noires " dont ils ignorent le fonctionnement, les chercheurs en sciences humaines et sociales sont plus que d'autres portés à imaginer l'informatique comme une discipline homogène et triomphante. Quoique la promotion hyperbolique oblitère l'histoire et la dissimule sous les promesses, l'ordinateur est un objet historique $^{16}$. Les études sur le hardware - les réalisations, parmi d'autres possibles, de la machine universelle conçue au milieu du $\mathrm{xx}^{\mathrm{e}}$ siècle - et sur le software se développent seulement maintenant, contre l'obsolescence des matériels et des programmes ${ }^{17}$. Si l'informatique est une aventure intellectuelle collective, comme toute science, son historicité est celle des groupes humains qui lui donnent ses formes et sont en même temps transformés par son usage ${ }^{\mathrm{I} 8}$.

Une histoire des humanités numériques n'a guère de sens si elle n'est à la fois scientifique, culturelle, institutionnelle et technologique. Comment produire collectivement une telle histoire? En profitant de l'ouverture d'un dialogue entre spécialités et en rassemblant les matériaux hérités sous bénéfice d'inventaire, pour commencer.

Ce numéro spécial de Critique inscrit l'évolution des pratiques savantes dans le contexte plus large d'un changement culturel. Cela ne signifie pas que l'on puisse dissoudre les problèmes scientifiques et institutionnels dans une conception générale. Les humanités numériques sont peut-être un moment, mais ce moment est lourd d'enjeux. Au fil des décennies, ce champ peut

University Press, Amsterdam : I. Early Modern Europe, 2010; II. From Early Modern to Modern Disciplines, 2012 ; III. The Modern Humanities, 2014.

16. Michael Mahoney, "The Histories of Computing(s) ", Interdisciplinary Science Reviews, 30, 2, 2005, p. II9I35 (" hype hides history", p. I 20). Lire également Willard McCarty, "As It Almost Was : Historiography of Recent Things ", Literary and Linguistic Computing, I9, 2, 2004, p. I6I-I8I.

I7. Martin Campbell-Kelly, From Airline Reservations to Sonic the Hedgehog. A History of the Software Industry, Cambridge, Massachusetts Institute of Technology Press, 2003.

I8. Par contraste, l'un des principaux théoriciens de l'informatique s'est récemment fait l'avocat d'une histoire de sa discipline faite de témoignages personnels et d'une forte documentation technique : Donald Knuth, "Let's Not Dumb Down the History of Computer Science ", 20I4 Kailath Lecture, 20I4, http://kailathlecture.stanford.edu/ 20 I 4 KailathLecture.html. 
demeurer comme un épicentre de l'expérimentation ou une zone d'échange. Cependant, quels que soient leurs noms et leurs métamorphoses à venir, les humanités numériques ont des futurs contingents. C'est notre responsabilité commune, au cours de la naturalisation de certains de leurs aspects dans les disciplines existantes, de ne pas inhiber le ferment d'innovation et de critique qu'elles représentent. La dimension rétrospective que nous avons évoquée fait partie de leur apport : par leur réflexivité technique et méthodologique, elles incitent à approfondir les histoires des pratiques de recherche et d'enseignement. En ce sens, les humanités numériques ne sont pas seulement une boîte à outils et un espace de dialogue, mais aussi un bon objet pour les sciences humaines et sociales.

Aurélien Berra 